\title{
Fitzpatrick Skin Type I
}

National Cancer Institute

\section{Source}

National Cancer Institute. Fitzpatrick Skin Type I. NCI Thesaurus. Code C74569.

Skin which is extremely pale white and it always burns when exposed to the sun. It is seen in people with blond or red hair and blue or hazel eyes. 